Article

\title{
Smart Card-Based Secure Authentication Protocol in Multi-Server IoT Environment
}

\author{
Won-il Bae 1, Jin Kwak 2,* \\ 1 Department of Computer Engineering, Ajou University, Suwon 16499, Korea; wibae.isaa@gmail.com \\ 2 Department of Cyber Security, Ajou University, Suwon 16499, Korea \\ * Correspondence: security@ajou.ac.kr;
}

\begin{abstract}
In recent years, the internet of things has been widely utilized in various fields, such as in smart factories or connected cars. As its domain of application has expanded, it has begun to be employed using multi-server architectures for a more efficient use of resources. However, because users wishing to receive IoT services connect to multi-servers over wireless networks, this can expose systems to various attacks and result in serious security risks. To protect systems (and users) from potential security vulnerabilities, a secure authentication technology is necessary. In this paper, we propose a smart card-based authentication protocol, which performs the authentication for each entity by allowing users to go through the authentication process using a smart card transmitted from an authentication server, and to login to a server connected to the IoT. Furthermore, the security of our proposed authentication protocol is verified by simulating a formal verification scenario using AVISPA, a security protocol-verification tool.
\end{abstract}

Keywords: user authentication; multi server; internet of things; formal verification; security

\section{Introduction}

By enabling devices such as machines to exchange information with embedded software, sensors, and so on via internet networks and consequently enhancing the functionality and performance of individual devices, the IoT(Internet of Things) enables the provision of new intelligent services. In recent years, as the applications of the IoT have expanded, it has undergone considerable progress through interactions with a diverse range of other industries, and has been employed in various applications such as smart factories, intelligent buildings, and connected cars [1].

Although the IoT is utilized in various fields, the performance of its sensors is not particularly high. Therefore, it is employed with multi-servers, in order to increase the resource efficiency. In particular, in terms of reducing the number of operations, the authentication protocol of a smart cardbased multi-server environment involves the operations of one-way functions and the exclusive or (XOR) function. Thus, this authentication protocol has been the subject of ongoing research [2-6].

However, if the multi-server authentication system is vulnerable, then attacks such as user impersonation, session key leakages, and replay attacks may occur in the process of connecting to the multi-server where the user stores IoT information over wireless networks [7]. This may result in leakages of confidential information by an unauthorized attacker, data sniffing and forgery/tampering, and other attacks during the communication process, and may lead to issues with the service availability of the multi-server [8-10]. Therefore, to mitigate the potential security vulnerabilities in a multi-server IoT environment, a secure authentication protocol is required.

In this study, we analyze the threats that may occur in multi-server IoT environment net works during the communication process, and propose a secure authentication protocol that $\mathrm{c}$ an respond to such security threats. In addition, we verify the security of the proposed protoc 
ol by performing a formal verification of the proposed authentication protocol using the AVIS PA(Automated Validation of Internet Security Protocols and Applications) 1 .

In the authentication protocol proposed in this paper, a user logs in to the IoT server using a smart card, and the authentication server can verify the user and the IoT server. By generating the same session key, the authentication process between the user, the IoT server, and the authentication server is performed. The composition of this paper is as follows.

In Section 2, we describe the security threats that may occur in a multi-server IoT environment and explain AVISPA, a formal verification tool for security protocols. In Section 3, we propose a secure authentication protocol for multi-server IoT environments, and describe the authentication protocol specified by the HLPSL(high-level protocol specification language). In Section 4, we describe the security analysis of the proposed protocol, and in Section 5, we simulate the formal verification of the authentication protocol via AVISPA. Finally, our conclusions are listed in Section 6.

\section{Related work}

\subsection{Multi-server IoT security threats}

The vast amount of data collected by sensors connected to a multi-server can be considered an attractive target for attackers. In a multi-server IoT environment, a user may access the multi-server over a wireless network, which can be a serious security threat. The security threats that can occur in the network communication process of a multi-server IoT environment are as follows [11,12].

- User impersonation attack

In this kind of attack, assuming that the malicious attacker has knowledge of the user's login request message from the previous session with the IoT server, the attacker masquerades as the authorized user by deriving the login request message of the current session.

- Session key disclosure attack

An public channel can occur when a user connects to the server over a wireless network. In this public channel, a malicious attacker can leak the session key by extracting the secret values. Through this attack, leakage and forgery/tampering of data stored on the server can occur.

- Denial-of-service attack

If one or more attackers generate a large number of identical login request messages using their smart cards and send them to the server connected to the sensor, then there may be a problem in service availability in the server.

- Replay attack

In this type of attack, an attacker can authenticate the user from the server connected to the sensor by storing the message that was communicated to the authentication server in the previous session for the authenticated user, and retransmitting the message to the current session or a subsequent session.

- $\quad$ Server spoofing attack

A malicious attacker can impersonate the IoT server when the user logs in. Therefore, the attacker can masquerade as the server to obtain the user login information.

1 AVISPA, DIST, Eidgenoessische Technische Hochschule Zuerich (ETHZ), CASSIS, Siemens Aktiengesellschaft, http://www.avispa-project.org/ 
- Invasion of privacy

Invasion of privacy is a violation of privacy through the revelation of private material, exposing various information and communication subjects of the user on the communication networks between the user and the server.

\subsection{AVISPA}

AVISPA is a tool that formally verifies the security of the internet protocol, and notifies $t$ he user with messages when it discovers attacks against the protocol [13]. AVISPA is compos ed of independently developed modules and the HLPSL, which is used as the input for the $p$ rotocol specification. HLPSL is a module-type role-based language that can express various op erators, as well as data flow and structure, intruder models, and complex security properties [ 14].

The roles can be divided into two types according to their purposes. One type consists of descriptions of the values required to describe each entity, and performs message transmission/reception using SND and RCV commands. The other role is to contain the overall scenario, and include the contents of the declared constants, the information known to an attacker, and the verification properties for the authentication protocol.

Furthermore, AVISPA is automatically generated in intermediate format (IF) via the HLPSL2IF translator, and used as input to the OFMC(On-the-fly Model-Checker), CL-AtSe(CL-based Attack Searcher), SATMC(SAT-based Model-Checker), and TA4SP(Tree Automata-based Protocol Analyser) models. The schematic of its architecture is shown in Figure 1 [15].

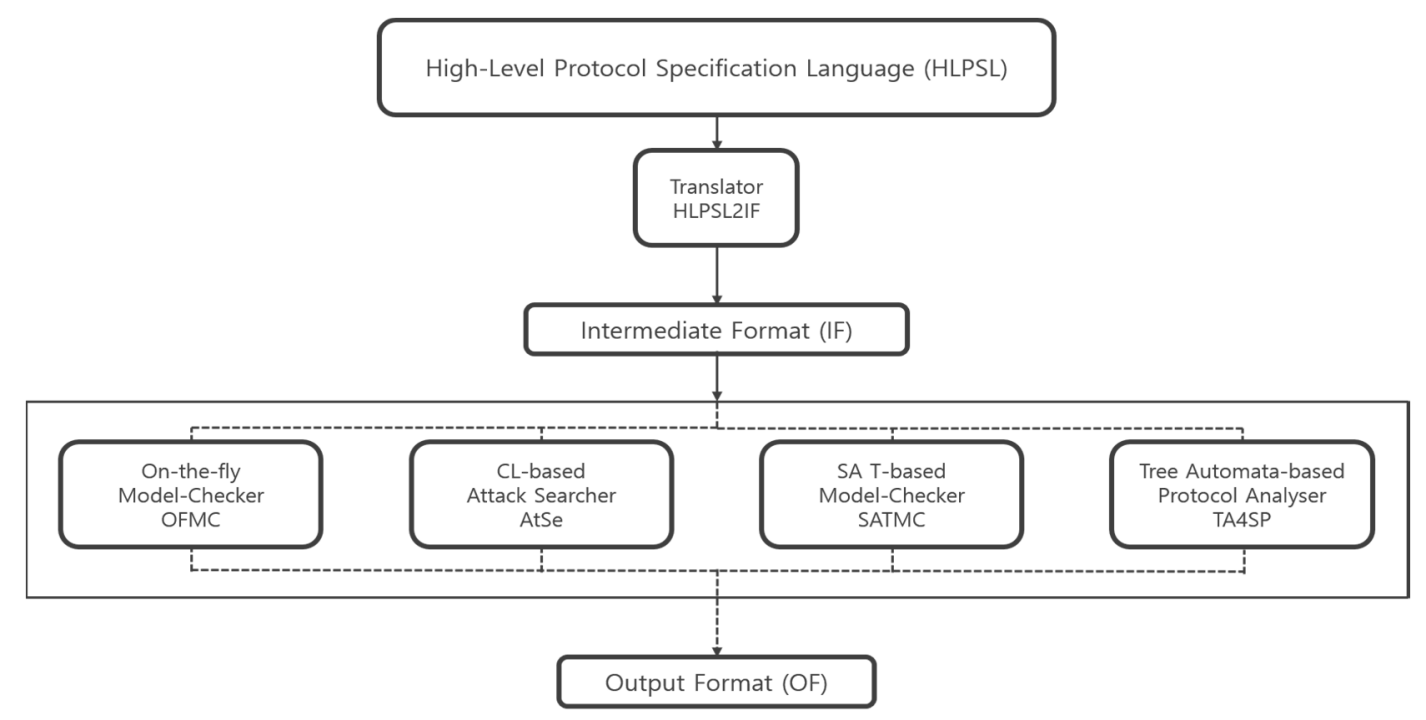

Figure 1. Architecture of the AVISPA tool

\section{Proposed authentication protocol}

The authentication protocol proposed in this paper consists of the user $\boldsymbol{U}_{\boldsymbol{i}}$, IoT server $\boldsymbol{S}_{\boldsymbol{j}}$, and authentication server $\boldsymbol{C S}$. When the user logs into the IoT server, the protocol performs authentication for each entity. Table 1 lists the parameter values used in the proposed authentication protocol. 
Table 1. Notation used in the proposed protocol

\begin{tabular}{|c|c|}
\hline Notation & Description \\
\hline$U_{i}$ & i th user \\
\hline$S_{j}$ & $\mathrm{j}$ th user \\
\hline$C S$ & Control server \\
\hline$I D_{\boldsymbol{i}}$ & Identity of $U_{i}$ \\
\hline$P_{i}$ & Password of $U_{i}$ \\
\hline$U I D_{i}$ & Anonymity value of $U_{i}$ \\
\hline$S I D_{j}$ & Identity of $S I D_{j}$ \\
\hline$x$ & Master secret key chosen by CS \\
\hline$T s$ & Timestamp \\
\hline$N_{i 1}$ & $\begin{array}{l}\text { Random number generated by } U_{i}{ }^{\prime} \text { s } \\
\text { smart card for session key } \\
\text { agreement }\end{array}$ \\
\hline$N_{i 2}$ & $\begin{array}{l}\text { Random number generated by } \\
\mathrm{S}_{\mathrm{j}} \text { for session key agreement }\end{array}$ \\
\hline$N_{i 3}$ & $\begin{array}{l}\text { Random number generated by CS } \\
\text { for session key agreement }\end{array}$ \\
\hline$S K$ & $\begin{array}{l}\text { Common session key shared } \\
\text { among } U_{i}, \mathrm{~S}_{\mathrm{j}} \text {, and CS }\end{array}$ \\
\hline$h\left({ }^{*}\right)$ & $\begin{array}{l}\text { Collision-free one-way hash } \\
\text { function }\end{array}$ \\
\hline$\oplus$ & Exclusive OR operation \\
\hline$\|$ & Message concatenation operation \\
\hline
\end{tabular}

There are three phases in the proposed authentication protocol, and these are carried out in the following order: registration, login and authentication, and password change. CS is an authentication server that can be trusted, and it is responsible for the registration and authentication of the user and the IoT server. In addition, because the proposed authentication protocol employs timestamps, the authentication server $C S$, user $U_{i}$ and IoT server $S_{j}$ performs time synchronization.

\subsection{Registration phase}

During the registration phase, the user $U_{i}$ and the IoT server $S_{j}$ request registration to the authentication server CS. In return, the authentication server issues a smart card to the user $U_{i}$, and sends the necessary values for the login and authentication phases to the IoT server $S_{j}$. This process is illustrated in Figure 2.

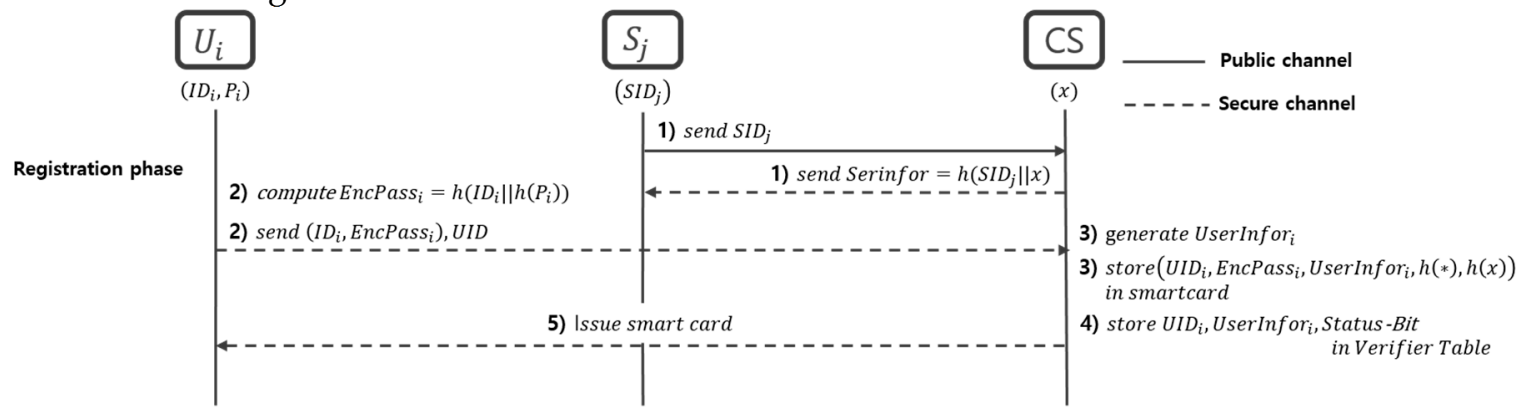

Figure 2. Registration phase 
STEP 1. $S_{j}$ sends its identification value $S I D_{j}$ to $C S$ via a secured channel, and $C S$ computes the Serinfor $r_{j}$ value that contains the information on the IoT server send to $S_{j}$ via secure channel.

$$
\text { Serinfor }{ }_{j}=h\left(\operatorname{SID}_{j} \| x\right)
$$

STEP 2. $U_{i}$ chooses the user $I D_{i}$ and password $P_{i}$, computes EncPass $s_{i}$, and sends the registration request message $\left(I D_{I}, \operatorname{EncPass}_{i}, U I D_{i}\right)$ with the user's anonymity values to $C S$.

$$
\text { EncPass }_{i}=h\left(I D_{i} \| h\left(P_{i}\right)\right)
$$

STEP 3. CS generates the user's secret information value, Userinfor ${ }_{i}$, and stores $U I D_{i}$, Userinfor $_{i}$, EncPass $_{i}, h(*)$, and $h(x)$ in the smart card.

$$
\text { Userinfor }_{i}=h\left(\text { EncPass }_{i} \| x\right)
$$

STEP 4. CS stores the user secret information value Userinfor $_{i}$, the user's anonymity value $U I D_{i}$, and the status-bit value in the verifier table. If the user performs the registration process, then the status-bit value is stored as 1 , and if there is no registration then the value is stored as 0 . The verifier table is presented in Table 2 .

Table 2. The verifier table

\begin{tabular}{ccc}
\hline $\begin{array}{c}\text { anonymity } \\
\text { value }\end{array}$ & User-verifier & Status-bit \\
\hline$\vdots$ & $\vdots$ & $\vdots$ \\
$U I D_{i}$ & Userinfor $_{i}$ & $0 / 1$ \\
$U I D_{i}$ & Userinfor $_{j}$ & $0 / 1$ \\
$\vdots$ & $\vdots$ & $\vdots$ \\
\hline
\end{tabular}

STEP 5. CS issues the smart card to the user $U_{i}$.

\subsection{Login and authentication phase}

During the login and authentication phase, the verification of the legitimate smart card holder is performed. In order to login, the user $U_{i}$ sends a login request message to the IoT server $S_{j}$, and $C S$ performs the verification of each entity. Then, $U_{i}, S_{j}$, and $C S$ all generate the same session key (Figure 3). 


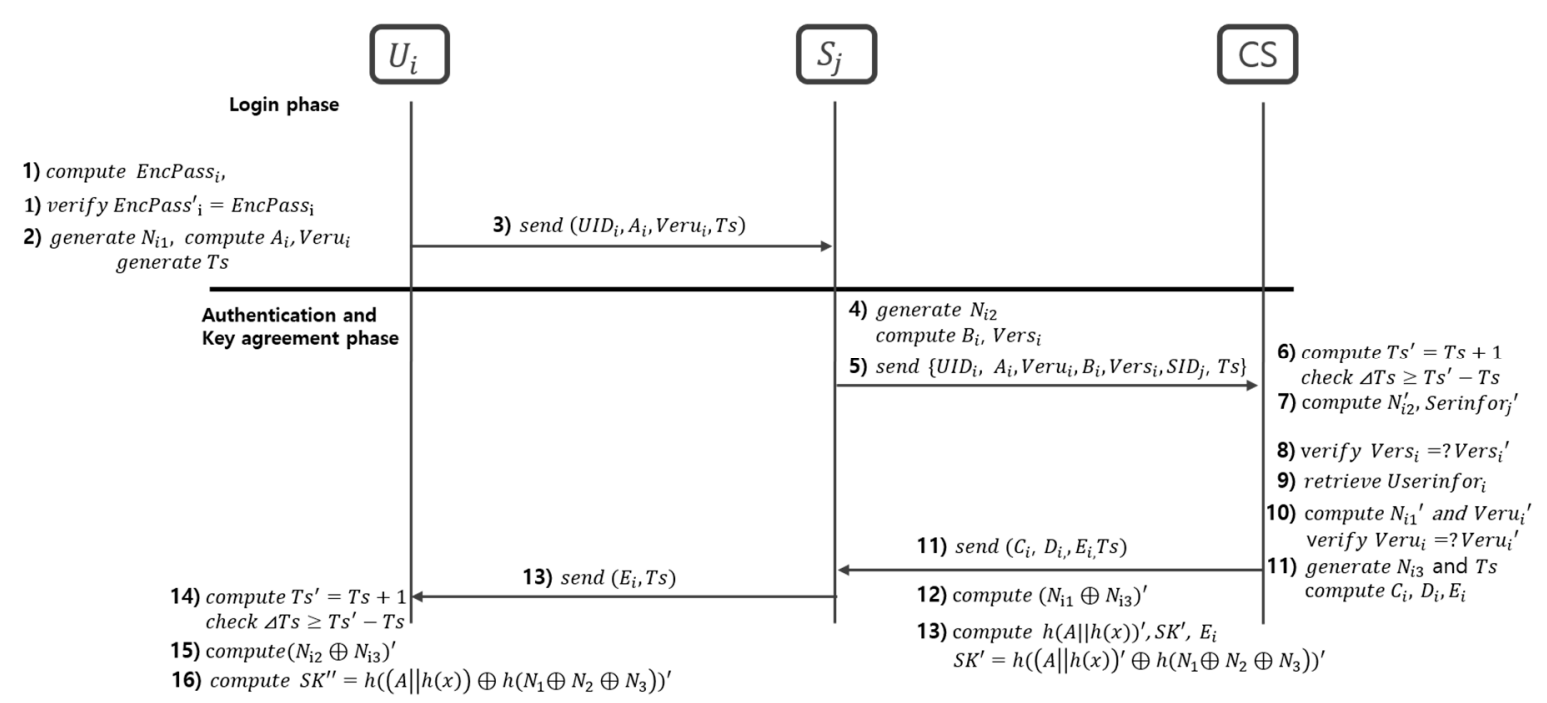

Figure 3. Login and authentication phase

STEP 1. $U_{i}$ inserts the smart card into the card reader, and enters their ID, $I D_{i}$, and password, $P_{i}$. The smart card computes EncPass ${ }_{i}{ }^{\prime}$, and compares the information with EncPass ${ }_{i}$ contained in the smart card. If the information matches, then the user is verified as the legitimate owner of the smart card. If the information does not match, then the session is terminated.

$$
\begin{gathered}
\text { EncPass }_{i}{ }^{\prime}=h\left(I D_{i} \| h\left(P_{i}\right)\right) \\
\text { EncPass }_{i}=\text { = EncPass }{ }_{i}{ }^{\prime}
\end{gathered}
$$

STEP 2. The user $U_{i}$ who is verified as the legitimate owner of the smart card, selects a random value $N_{j 1}$ to be generated for each session, and computes $A_{i}$ and Veru $u_{i}$ with $h(x)$ and UserInfor $i$ contained in the smart card and the chosen random value $N_{j 1}$. Then, the timestamp Ts is generated.

$$
\begin{gathered}
A_{i}=U_{\text {serinfor }} \oplus h(x) \oplus N_{i 1} \\
\operatorname{Veru}_{i}=h\left(h(x) \| N_{i 1}\right)
\end{gathered}
$$

STEP 3. The user $U_{i}$ configures the login request message $\left(A_{i}, V e r u_{i}, U I D_{i}, T s\right)$ with his/her anonymity value $U I D_{i}$, computes $A_{i}$ and $T s$, and sends the message to the IoT server $S_{j}$.

STEP 4. The IoT server $S_{j}$ that received the login request message from $U_{i}$ selects a random value $N_{i 2}$ to be generated for each session, and computes $B_{i}$ and $\operatorname{Vers}_{i}$ using the Serinfor ${ }_{j}$ value received in the registration phase.

$$
\begin{gathered}
B_{i}=\text { Serinfor }_{j} \oplus N_{i 2} \\
\operatorname{Vers}_{i}=h\left(h(x) \| N_{i 2}\right)
\end{gathered}
$$

STEP 5. $S_{i}$ sends the login request message $\left(U I D_{i}, A_{i}, \operatorname{Veru}_{i}, B_{i}, \operatorname{Vers}_{i}, \operatorname{SID}_{j}, T s\right)$ to CS. The message is configured for the $A_{i}, U I D_{i}$ (received from the user $U_{i}$ ), $S_{i}$ 's own identification value $S I D_{j}, B_{i}$ (which was generated earlier), and the timestamp Ts.

STEP 6. The CS that received the login request message from $S_{i}$ computes $T s^{\prime}=T s+1$, and then confirms whether $\Delta T s \geq T s^{\prime}-T s$. Here, $T s^{\prime}$ is the stamp for the time the server received the login 
message, and $\Delta T s$ is the minimum authentication time considering the time for the login message transmission.

STEP 7. CS generates Serinfor ${ }_{j}{ }^{\prime}$ using the received $S I D_{j}$ value and its own master key, and extracts the $N_{i 2}$ value via the $B_{i}$ value received from the login request message.

$$
\begin{gathered}
\text { Serinfor }_{j}{ }^{\prime}=h\left(\operatorname{SID}_{j} \| x\right) \\
{N_{i 2}}^{\prime}=\text { Serinfor }_{j}{ }^{\prime} \oplus B_{i}
\end{gathered}
$$

STEP 8. By using the computed $N_{i 2}{ }^{\prime}$ value, $C S$ generates the $V e r s_{i}{ }^{\prime}$ value, and if this matches the $B_{i}$ value received by the login request message, it is authenticated as the legitimate IoT server $S_{j}$; if not, the session is terminated.

$$
\begin{gathered}
\operatorname{Vers}_{i}{ }^{\prime}=h\left(h(x) \| N_{i 2}{ }^{\prime}\right) \\
\operatorname{Vers}_{i}=\operatorname{Vers}_{i}{ }^{\prime}
\end{gathered}
$$

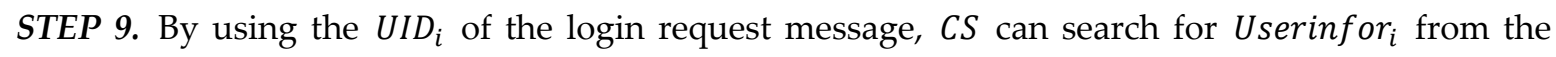
verifier table generated in the registration phase.

STEP 10. CS computes the $N_{i 1}^{\prime}$ value using the received $A_{i}$ value, the generated $h(x)$, and the previously retrieved Userinfor ${ }_{i}$. Using this computed $N_{i 1}^{\prime}$ value and $h(x)$, it generates the $\operatorname{Veru}_{i}{ }^{\prime}$ value. Next, if this matches the $\operatorname{Veru}_{i}$ value received with the login request message, then it is authenticated as a legitimate user, and generates the following session key $S K$. If it does not match, then the session is terminated.

$$
\begin{gathered}
N_{i 1}^{\prime}=\text { Userinfor }_{i} \oplus h(x) \oplus A_{i} \\
\text { Veru }_{i}{ }^{\prime}=h\left(h(x) \| N_{i 1}{ }^{\prime}\right) \\
\text { Veru }_{i}={\text { ? } \text { Veru }_{i}{ }^{\prime}} \\
S K_{i}=h\left(h(A \| h(x)) \oplus h\left(N_{i 1} \oplus N_{i 2} \oplus N_{i 3}\right)\right)
\end{gathered}
$$

STEP 11. The $C S$ generates the timestamp Ts and selects the random value $N_{i 3}$. Next, it computes $C_{i}, D_{i}$, and $E_{i}$, and sends the mutual authentication message $\left(C_{i}, D_{i}, E_{i}, T s\right)$ to $S_{i}$.

$$
\begin{gathered}
C_{i}=N_{\mathrm{i} 1} \oplus N_{\mathrm{i} 3} \oplus h\left(S I D_{j} \oplus N_{\mathrm{i} 2}\right) \\
D_{i}=h(A \| h(x)) \oplus h\left(S I D_{j} \oplus N_{\mathrm{i} 2}\right) \\
E_{i}=N_{\mathrm{i} 2} \oplus N_{\mathrm{i} 3} \oplus h(A \| h(x))
\end{gathered}
$$

STEP 12. $S_{i}$ receives the mutual authentication message, and computes $\left(N_{i 1} \oplus N_{i 3}\right)^{\prime}$ via its own $S I D_{j}$ value and the random value $N_{i 2}$.

$$
\left(N_{\mathrm{i} 1} \oplus N_{\mathrm{i} 3}\right)^{\prime}=C_{i} \oplus h\left(S I D_{j} \oplus N_{\mathrm{i} 2}\right)
$$

STEP 13. $S_{i}$ computes $h(A \| h(x))^{\prime}$ via its own $S I D_{j}$ value and the random value $N_{i 2}$, using the $D_{i}$ value received in the mutual authentication message. It generates the session key $S K$ by on operating 
its own random value $N_{i 2}$ with the previously computed $\left(N_{i 1} \oplus N_{i 3}\right)^{\prime}$. Next, $S_{i}$ computes $E_{i}$ and sends a login response message $\left(E_{i}, T s\right)$ to the user $U_{i}$.

$$
\begin{gathered}
h(A \| h(x))^{\prime}=D_{i} \oplus h\left(S I D_{j} \oplus N_{\mathrm{i} 2}\right) \\
S K^{\prime}=h\left(h(A \| h(x))^{\prime} \oplus h\left(N_{\mathrm{i} 1} \oplus N_{\mathrm{i} 2} \oplus N_{\mathrm{i} 3}\right)\right)^{\prime} \\
E_{i}=\left(N_{\mathrm{i} 2} \oplus N_{\mathrm{i} 3}\right) \oplus h(A \| h(x))
\end{gathered}
$$

STEP 14. After receiving the login request message from the $S_{i}$, the user $U_{i}$ computes $T s^{\prime}=T s+1$ to confirm that $\Delta T s \geq T s^{\prime}-T s . T s^{\prime}$ is the timestamp for when the login message is received by the server, and $\Delta T s$ is the minimum authentication time considering the transmission time for the login message.

STEP 15. By using the $E_{i}$ value received in the mutual authentication message, $U_{i}$ can compute the $\left(N_{i 2} \oplus N_{i 3}\right)^{\prime}$ value via the $A_{i}$ value generated by the user and the $h(x)$ value contained in the smart card.

$$
\left(N_{\mathrm{i} 2} \oplus N_{\mathrm{i} 3}\right)^{\prime}=E_{i} \oplus h(A|| h(x))
$$

STEP 16. $U_{i}$ can operate on its own random value $N_{i 1}$ with $\left(N_{2} \oplus N_{3}\right)^{\prime}$ that was computed earlier, and can generate the session key $S K$ via its own $A_{i}$ value and the $h(x)$ value contained in the smart card. Therefore, the user $U_{i}$, IoT server $S_{j}$, and authentication server $C S$ can perform the authentication by generating the same session key.

$$
S K^{\prime \prime}=h(A \| h(x)) \oplus\left(N_{\mathrm{i} 1} \oplus N_{\mathrm{i} 2} \oplus N_{\mathrm{i} 3}\right)^{\prime}
$$

\subsection{Password change phase}

The password change phase is the process performed when the user $U_{i}$ wants to change their password $P_{i}$ to a new one $P_{i}{ }^{N E W}$. The process is illustrated in Figure 4.

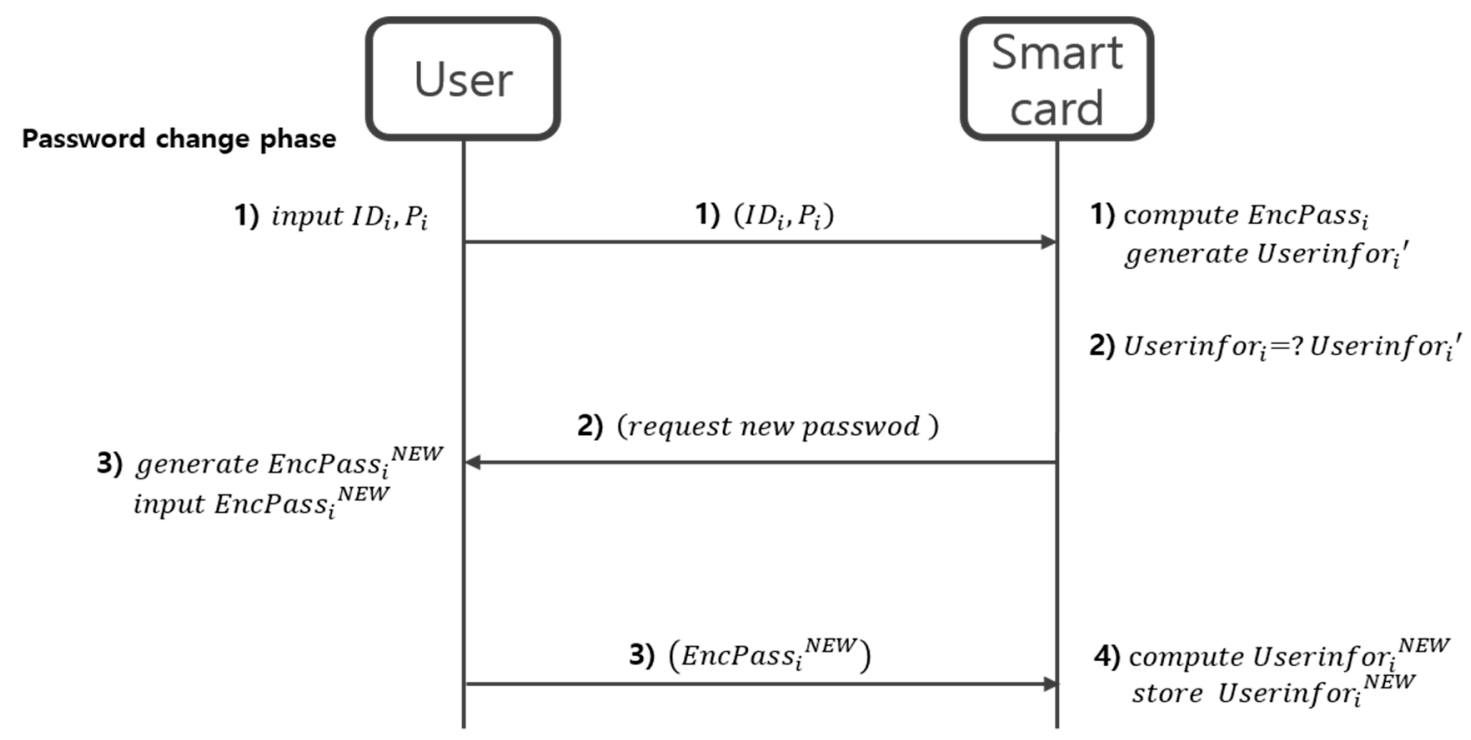

Figure 4. Password change phase

STEP 1. The user $U_{i}$ inserts a smart card into the card reader and inputs their ID, $I D_{i}$, and password, $P_{i}$. The smart card computes EncPass $i$ and generates Userinfor $_{i}{ }^{\prime}$ using the computed EncPass ${ }_{i}$. 


$$
\begin{aligned}
\operatorname{EncPass}_{i} & =h\left(I D_{i} \| h\left(P_{i}\right)\right) \\
\text { Userinfor }_{i}{ }^{\prime} & =h\left(\text { EncPass }_{i} \| x\right)
\end{aligned}
$$

STEP 2. The smart card compares the generated $U_{\text {serinfor }}{ }^{\prime}$ with $U_{\text {serinfor }}$ contained in the smart card. If these match, then the user is verified as the legitimate owner of the smart card, and the user can change the password. The smart card requests a new password.

$$
\text { Userinfor }_{i}=\text { ? Userinfor }{ }_{i}^{\prime}
$$

STEP 3. Once verified as the legitimate smart card owner, the user enters the new password $P_{i}{ }^{N E W}$, generates EncPass $_{i}{ }^{N E W}$, and enters this into the smart card.

$$
\operatorname{EncPass}_{i}{ }^{N E W}=h\left(I D_{i} \| h\left(P_{i}^{N E W}\right)\right)
$$

STEP 4. By using the EncPass ${ }_{i}{ }^{N E W}$ generated in the above process, the new Userinfor $_{i}{ }^{N E W}$ can be computed, and the existing Userinfor ${ }_{i}$ is replaced with Userinfor $_{i}{ }^{N E W}$, which is stored in the smart card. In this manner, the password change process is completed.

$$
\text { Userinfor }_{i}^{N E W}=h\left(\text { EncPass }_{i}{ }^{N E W} \| x\right)
$$

\subsection{Authentication protocol specification utilizing HLPSL.}

In this study, we use the AVISPA web tool for the formal verification of the authentication protocol. The AVISPA web tool can specify the authentication protocol with the CAS and HLPSL languages. This section explains the registration, and login and authentication phases of the proposed authentication protocol written in the HLPSL language.

Because AVISPA is a role-based language, it assigns a role to each participant. It is composed of role_U, which represents the user, role_S, which is assigned to the IoT server, and role_CS, representing the authentication server. In the role environment (), the constants used by the specified protocol are defined, and can specify the property values known to the attacker. In addition, secrecy_of and weak_authentication_on are specified depending on the goal, in order to add the secrecy () and witness () functions. These functions are used to verify the security and authentication. The security and authentication are the properties of the protocol's verification.

In the part where the formula is specified, concatenation can be expressed as ".", xor operates as "xor(A, B)," and the exponent $E^{N}$ operates as "exp(E, N)." In addition, in the part where each role is specified, the message specified as RCV can be transmitted, and the received contents can be expressed as SND.

This section deals with the specifications for the sent and received messages, and for the security properties. Table 3 details the specification of the user, and Table 4 concerns the specification of the IoT server. Table 5 presents the specification of the authentication server, and Table 6 illustrates the role environment, which contains the specified constants. Finally, Table 7 shows the specification of the goal, by specifying a function to verify the authentication protocol. 
Table 3. Specification for the User

transition

3. State $=0 /$ RCV(start $)=\mid>$ State': $=1 /$ Key_1':=new ()$/$

Key_set_U_CS':=cons(Key_1',Key_set_U_CS)

$\bigwedge \mathrm{SND}\left(\{\mathrm{ID}, \mathrm{H}(\mathrm{ID} . \mathrm{H}(\mathrm{P})), \mathrm{UId}\}_{-} \mathrm{Key}_{-} 1 '\right)$

4. State $=1 /$ in $($ Key_2',Key_set_CS_U)

$\bigwedge \mathrm{RCV}\left(\left\{\mathrm{UId}, \mathrm{H}(\mathrm{H}(\mathrm{X}) \cdot \mathrm{NU}), \mathrm{H}(\mathrm{ID} \cdot \mathrm{H}(\mathrm{P})), \mathrm{H}\left(\mathrm{H}(\mathrm{ID} \cdot \mathrm{H}(\mathrm{P})) . \mathrm{X}^{\prime}\right)\right\}_{-} \mathrm{Key}_{2} 2^{\prime}\right)=\mid>$

State':=2 $\bigwedge$ Key_set_CS_U':=delete(Key_2',Key_set_CS_U) $/$ NU':=new ()

10. State $=2 / 入$

$\bigwedge \mathrm{SND}\left(\mathrm{UId}, \operatorname{xor}\left(\operatorname{xor}\left(\mathrm{H}\left(\mathrm{H}(\mathrm{ID} . \mathrm{H}(\mathrm{P})) . \mathrm{X}^{\prime}\right), \mathrm{H}\left(\mathrm{X}^{\prime}\right)\right), \mathrm{NU}\right)\right)$

RCV(xor(xor(NS',NCS'),H(xor(xor(H(H(ID.H(P)).X),H(X)),NU).H(X)))) =| > State':=3

$\wedge$

witness(CS,S,auth_14,xor(xor(NS',NCS'),H(xor(xor(H(H(ID.H(P)).X),H(X)),NU).H(

X))))

$\wedge \operatorname{secret}(\mathrm{NU}$, auth_10,\{S,U\})

end role

Table 4. Specification for the IoT server

transition

1. State $=0 / \triangle R C V($ start $)=\mid>$ State' $:=1$

$\bigwedge \mathrm{SND}(\mathrm{SId})$

2. State $=1$ / in $($ Key_1',Key_set_CS_S $) / \operatorname{RCV}\left(\left\{\mathrm{H}\left(\mathrm{SId} . \mathrm{X}^{\prime}\right)\right\} \_\right.$Key_1') $=\mid>$State':=2 八

Key_set_CS_S':=delete(Key_1',Key_set_CS_S)

5. State $=2 / \backslash \mathrm{RCV}\left(\mathrm{UId}^{\prime}, \mathrm{H}\left(\mathrm{H}(\mathrm{X}) \cdot \mathrm{NU} \mathrm{U}^{\prime}\right), \operatorname{xor}\left(\operatorname{xor}\left(\mathrm{H}\left(\mathrm{H}\left(\mathrm{ID}{ }^{\prime} \cdot \mathrm{H}\left(\mathrm{P}^{\prime}\right)\right) . \mathrm{X}\right), \mathrm{H}(\mathrm{X})\right), \mathrm{NU}^{\prime}\right)\right)=\mid>$ State':=3

$\wedge \operatorname{secret}\left(\mathbf{P}^{\prime}\right.$, auth_1, $\left.\{\mathrm{U}, \mathrm{S}\}\right)$

$\wedge \operatorname{secret}(\mathrm{H}(\mathrm{X})$,auth_2,\{U,S\})

$\wedge \operatorname{secret}\left(N^{\prime}\right.$, auth_3, $\left.\{\mathrm{U}, \mathrm{S}\}\right) \wedge \mathrm{NS}$ ':=new()

$\Lambda$

SND(UId',H(H(X).NU'),H(H(X).NS), xor(xor(H(H(ID'.H(P')).X),H(X)),NU'), xor(H(SId.X), NS'),SId)

7. State $=3 /$

RCV(xor(xor(NS,NCS'),H(xor(xor(H(H(ID.H(P)).X),H(X)),NU).H(X)))) = |> State':=4

$\wedge \operatorname{secret}\left(\mathrm{NCS}^{\prime}\right.$, auth_6, $\left.\{\mathrm{CS}, \mathrm{S}\}\right)$

$\wedge$ secret(NS,auth_7,\{CS,S\})

$\wedge \operatorname{secret}(\mathrm{NU}$, auth_8, $\{\mathrm{CS}, \mathrm{S}\})$

$\wedge \operatorname{secret}(\mathrm{H}(\mathrm{X})$,auth_9, $\{\mathrm{CS}, \mathrm{S}\})$

$\wedge$ witness(S,CS,auth_13, $\left.\operatorname{xor}\left(\operatorname{xor}\left(\mathrm{NU}^{\prime}, \mathrm{NCS}^{\prime}\right), \mathrm{H}\left(\operatorname{xor}\left(\mathrm{SId}, \mathrm{NS}^{\prime}\right)\right)\right)\right)$

8. State $=4 /$

$\mathrm{RCV}(\operatorname{xor}(\operatorname{xor}(\operatorname{xor}(\mathrm{H}(\mathrm{H}(\mathrm{ID} \cdot \mathrm{H}(\mathrm{P})) \cdot \mathrm{X}), \mathrm{H}(\mathrm{X})), \mathrm{NU}) \cdot \mathrm{H}(\mathrm{X}), \mathrm{H}(\operatorname{xor}(\mathrm{SId}, \mathrm{NS}))))=\mid>$ State $^{\prime}:=5$

9. State $=5 / \mathrm{RCV}\left(\operatorname{xor}\left(\operatorname{xor}\left(\mathrm{NU}^{\prime}, \mathrm{NCS}^{\prime}\right), \mathrm{H}\left(\operatorname{xor}\left(\mathrm{SId}, \mathrm{NS}^{\prime}\right)\right)\right)\right)=1>$ State': $=6$

$\bigwedge \mathrm{SND}(\operatorname{xor}(\operatorname{xor}(\mathrm{NS}, \mathrm{NCS}), \mathrm{H}(\operatorname{xor}(\operatorname{xor}(\mathrm{H}(\mathrm{H}(\mathrm{ID} \cdot \mathrm{H}(\mathrm{P})) . \mathrm{X}), \mathrm{H}(\mathrm{X})), \mathrm{NU}) . \mathrm{H}(\mathrm{X}))))$

end role 
Table 5. Specification for the authentication server

transition

1. State $=0$ 八 RCV(SId) $=\mid>$ State':=1 八 Key_1':=new ()$/$

Key_set_CS_S':=cons(Key_1',Key_set_CS_S $)$

$\bigwedge \mathrm{SND}\left(\{\mathrm{H}(\mathrm{SId} . \mathrm{X})\}_{-} \mathrm{Key} \_1^{\prime}\right)$

3. State $=1 /$ in $($ Key_2',Key_set_U_CS $)$

$\wedge$ RCV( $($ ID,H(ID.H(P')), UId' $\left.\} \_K e y \_2 '\right)=\mid>$ State':=2 /

Key_set_U_CS':=delete(Key_2',Key_set_U_CS) $/$ Key_3':=new ()$/ \triangle$

Key_set_CS_U':=cons(Key_3',Key_set_CS_U)

$\bigwedge \mathrm{SND}\left(\left\{\mathrm{UId}^{\prime}, \mathrm{H}(\mathrm{ID} \cdot \mathrm{H}(\mathrm{P})), \mathrm{H}\left(\mathrm{H}\left(\mathrm{ID} \cdot \mathrm{H}\left(\mathrm{P}^{\prime}\right)\right) . \mathrm{X}\right)\right\}_{-} \mathrm{Key}_{-} 3^{\prime}\right)$

6. State $=2 / 入$

RCV(UId,H(H(X).NU'),H(H(X).NS), $x$ or $\left(x o r(H(H(I D . H(P)) . X), H(X)), N U^{\prime}\right), x o r(H(S I d$.

$\left.\left.\mathrm{X}), \mathrm{NS}^{\prime}\right), \mathrm{SId}\right)=\mid>$ State':=3

$\wedge \operatorname{secret}(\mathrm{NS}$ ',auth_4, $\{\mathrm{S}, \mathrm{CS}\})$

$\wedge \operatorname{secret}\left(\mathrm{H}(\mathrm{SId} . \mathrm{H}(\mathrm{X}))\right.$, auth $\left.\_5,\{\mathrm{~S}, \mathrm{CS}\}\right)$

$\wedge$ witness(CS,S,auth_11,xor(H(SId.X),NS'))

$\wedge$ witness(CS,U,auth_12,xor(xor(H(H(ID.H(P)).X),H(X)),NU')) $\wedge$

NCS':=new()

$\mathrm{SND}\left(\operatorname{xor}\left(\operatorname{xor}\left(\mathrm{NS}^{\prime}, \mathrm{NCS}\right), \mathrm{H}(\operatorname{xor}(\operatorname{xor}(\mathrm{H}(\mathrm{H}(\mathrm{ID} \cdot \mathrm{H}(\mathrm{P})) \cdot \mathrm{X}), \mathrm{H}(\mathrm{X})), \mathrm{NU}) \cdot \mathrm{H}(\mathrm{X}))\right)\right)$

$\wedge \mathrm{SND}\left(\operatorname{xor}\left(\operatorname{xor}\left(\operatorname{xor}(\mathrm{H}(\mathrm{H}(\mathrm{ID} . \mathrm{H}(\mathrm{P})) . \mathrm{X}), \mathrm{H}(\mathrm{X})), \mathrm{NU}^{\prime}\right)\right.\right.$.

$\left.\left.\mathrm{H}(\mathrm{X}), \mathrm{H}\left(\operatorname{xor}\left(\mathrm{SId}, \mathrm{NS}^{\prime}\right)\right)\right)\right)$

$\bigwedge \mathrm{SND}\left(\operatorname{xor}\left(\operatorname{xor}\left(\mathrm{NU}^{\prime}, \mathrm{NCS}\right), \mathrm{H}\left(\operatorname{xor}\left(\mathrm{SId}, \mathrm{NS}^{\prime}\right)\right)\right)\right)$

end role

Table 6. Specification for the role environment

def $=$

role environment()

const

hash_0:function,

sid:text,

contr:agent,

man:agent,

password:text,

uid:text,

serv:agent,

secretvalue:text,

id:text,

auth_1, auth_2, auth_3, auth_4, auth_5, auth_6, auth_7, auth_8, auth_9, auth_10,

auth_11, auth_12, auth_13, auth_14:protocol_id

intruder_knowledge $=\{$ man, serv, contr,sid, uid,id $\}$

composition

session1(password,uid,man,serv,contr,secretvalue,sid,id, \{\}$,\{\},\{\}$ )

end role

Table 7. Specification for the goal 
goal

secrecy_of auth_1

secrecy_of auth_2

secrecy_of auth_3

secrecy_of auth_4

secrecy_of auth_5

secrecy_of auth_6

secrecy_of auth_7

secrecy_of auth_8

secrecy_of auth_9

secrecy_of auth_10

weak_authentication_on auth_11

weak_authentication_on auth_12

weak_authentication_on auth_13

weak_authentication_on auth_14

end goal

\section{Security analysis}

\subsection{User impersonation (i.e., masquerading) attack}

An attacker impersonates the authorized user by extracting the login request message of the current session, assuming that he/she knows the login request message from the previous session of the IoT server.

Assume that the attacker has learned the login message $\left(U I D_{i}, A_{i}, T s\right)$ of the previous session via the public channel. Then, because the malicious attacker cannot compute EncPass $s_{i}$, the random value $N_{i 1}$, and $h(x)$, they cannot compute $A_{i}$, which is composed of the Userinfor ${ }_{i} \oplus$ $h(x) \oplus N_{i 1}$ operation. Therefore, they cannot extract the login request message for the current session by impersonating the authorized user, and the proposed authentication protocol is secure against user impersonation attacks.

\subsection{Session key disclosure attack}

Assuming that an attacker can intercept and steal $A_{i}, B_{i}, C_{i}, D_{i}$, and $E_{i}$ through the previous session via public channels, they still cannot extract the session key. The attacker cannot compute Userinfor $_{i}$ and $N_{i 1}$ through the known value of $A_{i}$ because they cannot determine $h(X)$. Furthermore, the attacker cannot compute the value $N_{i 2}$ via the publicly accessible $B_{i}$ value, because they cannot determine Serinfor ${ }_{j}$. For the same reason, the attacker cannot extract the random values $N_{i 1}, N_{i 2}$, and $N_{i 3}$ selected by the user $U_{i}$, IoT server $S_{j}$, and the authentication server $C S$, respectively, from the published $C_{i}, D_{i}$, and $E_{i}$ values and $h(x)$ generated by the authentication server $C S$. Therefore, the proposed authentication protocol is secure against such attacks involving session key leakages.

\subsection{Denial-of-service (Dos) attack}

When an attacker uses their own smart card to send a large number of identical login request messages $\left(\mathrm{A}_{\mathrm{K} 1}, \mathrm{UID}_{\mathrm{K} 1}, \mathrm{Ts}\right),\left(\mathrm{A}_{\mathrm{K} 2}, \mathrm{UID}_{\mathrm{K} 2}, \mathrm{Ts}\right), \ldots,\left(\mathrm{A}_{\mathrm{Kn}}, \mathrm{UID}_{\mathrm{Kn}}, \mathrm{Ts}\right)$, the IoT server $S_{j}$ may experience problems with its availability. However, in the registration phase the value of the status bit is stored as 1, using the verifier table given in Table 2. In this manner, the proposed protocol is designed not 
to receive such login request messages. Thus, the proposed authentication protocol is secure against the denial-of-service attacks.

\subsection{Replay attack}

The proposed authentication protocol uses the timestamp for the authentication of messages in communications between the user $U_{i}$, the IoT server $S_{j}$, and the authentication server $C S$.

Therefore, if an attacker participates in a session to perform eavesdropping (i.e., sniffing) or forgery/tampering attacks on transmitted or received messages, the value of the timestamp Ts changes, and the protocol terminates the session.

\subsection{Server spoofing attack}

In a server spoofing attack, an attacker impersonates an IoT server to obtain the desired information. However, in the login phase of the proposed authentication protocol, the authenticated user is identified via the verification of the smart card as EncPass ${ }_{i}=$ ? EncPass ${ }_{i}{ }^{\prime}$. Because the session is terminated if this does not match, the attacker cannot masquerade as the IoT server. Thus, the proposed authentication protocol is secure against server spoofing attacks, because it verifies the IoT server $S_{i}$ by checking $\operatorname{Vers}_{i}=$ ? $\operatorname{Vers}_{i}{ }^{\prime}$ with the $C S$.

\subsection{Invasion of privacy}

Invasion of privacy is the infringement of privacy by exposing information regarding the subject during a communication procedure on a network. However, the proposed authentication protocol uses the identifiers of the user and the IoT server, $U I D_{i}$ and $S I D_{j}$, and the anonymity value, to perform the communication procedure. Therefore, when the transmitted/received messages on a network are thought to have been eavesdropped, the communication subject cannot be identified, thus ensuring the privacy of the user and the IoT server.

\section{Experimental result through formal verification}

In this section, we analyze the experimental results by employing AVISPA, a formal verification tool for the authentication protocol, where the registration phase and login and authentication phase are specified using the HLPSL language, as described in Section 3.4.

The result of executing the specified HLPSL code file using the AVISPA web tool is shown in Figure 5. From left to right, the figure shows $C S, S_{j}$, and $U_{i}$. The authentication messages are transmitted and received through eight main phases, including the registration phase. However, in practice, authentication messages are transmitted and received in 10 phases, because the $C_{i}, D_{i}$, and $E_{i}$ values sent to $S_{j}$ from $C S$ are divided and transmitted sequentially. 


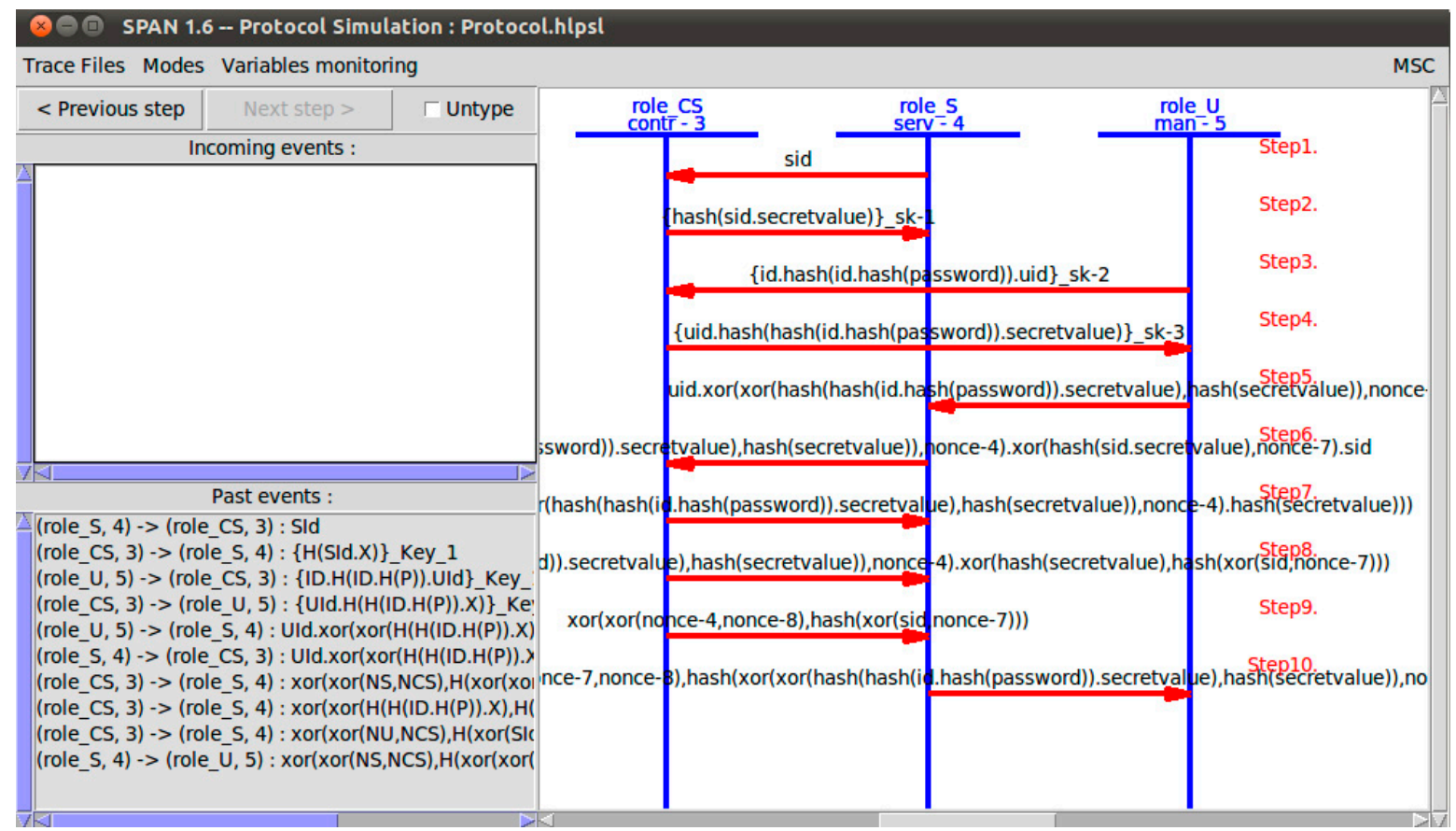

Figure 5. Execution screen of the AVISPA

The secret() and witness() functions and the verification properties for the authentication protocol were employed in the goal of the HLPSL created in Section 3.4 for verification. For the secret () and witness () functions shown in Table 7, the specified security properties are as follows:

1. $\quad \operatorname{secret}\left(\mathrm{P}^{\prime}\right.$, auth_1, $\left.\{\mathrm{U}, \mathrm{S}\}\right)$

This pertains to the login and authentication phase of STEP 3, and verifies whether the secrecy of $P_{i}$ in the login request message $\left(A_{i}, U I D_{i}, T s\right)$ between the user $U_{i}$ and IoT server $S_{i}$ is satisfied.

2. $\operatorname{secret}(\mathrm{H}(\mathrm{X})$, auth_2, $\{\mathrm{U}, \mathrm{S}\})$

This pertains to the login and authentication phase of STEP 3, and verifies whether the secrecy of $h(X)$ in the login request message $\left(A_{i}, U I D_{i}, T s\right)$ between the user $U_{i}$ and IoT server $S_{i}$ is satisfied.

3. $\quad$ secret(NU',auth_3,\{U,S\})

This pertains to the login and authentication phase of STEP 3, and verifies whether the secrecy of $N_{i 1}$ in the login request message $\left(A_{i}, U I D_{i}, T s\right)$ between the user $U_{i}$ and IoT server $S_{i}$ is satisfied.

4. $\quad$ secret(NS',auth_4, $\{\mathrm{S}, \mathrm{CS}\})$

This pertains to the login and authentication phase of STEP 5 , and verifies whether the secrecy of $N_{i 2}$ in the login request message $\left(\mathrm{UID}_{\mathrm{i}}, \mathrm{A}_{\mathrm{i}}, \operatorname{Veru}_{\mathrm{i}}, \mathrm{B}_{\mathrm{i}}, \operatorname{Vers}_{\mathrm{i}}, \mathrm{SID}_{\mathrm{j}}, \mathrm{Ts}\right)$ between the IoT server $S_{j}$ and the authentication server $C S$ is satisfied.

5. $\quad$ secret(H(SId.H(X)),auth_5, $\{S, C S\})$

This pertains to the login and authentication phase of STEP 5 , and verifies whether the secrecy of Serinfor ${ }_{j}$ in the login request message $\left(\mathrm{UID}_{\mathrm{i}}, \mathrm{A}_{\mathrm{i}}, \mathrm{Veru}_{\mathrm{i}}, \mathrm{B}_{\mathrm{i}}, \mathrm{Vers}_{\mathrm{i}}, \mathrm{SID}_{\mathrm{j}}, \mathrm{Ts}\right.$ ) between the IoT server $S_{j}$ and the authentication server $C S$ is satisfied. 
6. $\quad$ secret(NCS', auth_6, $\{\mathrm{CS}, \mathrm{S}\})$

This pertains to the login and authentication phase of STEP 11, and verifies whether the secrecy of $N_{i 3}$ in the mutual authentication message $\left(\mathrm{C}_{\mathrm{i}}, \mathrm{D}_{\mathrm{i}}, \mathrm{E}_{\mathrm{i}}, \mathrm{Ts}\right)$ between the authentication server $C S$ and the IoT server $S_{i}$ is satisfied.

7. $\quad$ secret(NS, auth_7, $\{\mathrm{CS}, \mathrm{S}\})$

This pertains to the login and authentication phase of STEP 11, and verifies whether the secrecy of $N_{i 2}$ in the mutual authentication message $\left(\mathrm{C}_{\mathrm{i}}, \mathrm{D}_{\mathrm{i}}, \mathrm{E}_{\mathrm{i}}, \mathrm{Ts}\right)$ between the authentication server $C S$ and the IoT server $S_{i}$ is satisfied.

8. $\quad \operatorname{secret}(\mathrm{NU}$, auth_8, $8 \mathrm{CS}, \mathrm{S}\})$

This pertains to the login and authentication phase of STEP 11, and verifies whether the secrecy of $N_{i 1}$ in the mutual authentication message $\left(\mathrm{C}_{\mathrm{i}}, \mathrm{D}_{\mathrm{i}}, \mathrm{E}_{\mathrm{i}}, \mathrm{Ts}\right)$ between the authentication server $C S$ and IoT server $S_{i}$ is satisfied.

9. $\quad \operatorname{secret}(\mathrm{H}(\mathrm{X})$, auth_9, $\{\mathrm{CS}, \mathrm{S}\})$

This pertains to the login and authentication phase of STEP 11, and verifies whether the secrecy of $h(X)$ in the mutual authentication message $\left(\mathrm{C}_{\mathrm{i}}, \mathrm{D}_{\mathrm{i}}, \mathrm{E}_{\mathrm{i}}, \mathrm{Ts}\right)$ between the authentication server $C S$ and the IoT server $S_{i}$ is satisfied.

10. $\operatorname{secret}(\mathrm{NU}$, auth_10, $\{\mathrm{S}, \mathrm{U}\})$

This pertains to the login and authentication phase of STEP 13, and verifies whether the secrecy of $N_{i 1}$ in the login response message $\left(\mathrm{E}_{\mathrm{i}}, \mathrm{Ts}\right)$ between the IoT server $S_{i}$ and the user $U_{i}$ is satisfied.

11. witness(CS,S,auth_11,xor(H(SId.X),NS'))

This pertains to the login and authentication phase of STEP 8, and the authentication server $C S$ verifies whether the IoT server $S_{j}$ is authenticated through the Vers $s_{i}$ value in the login request message $\left(\mathrm{UID}_{\mathrm{i}}, \mathrm{A}_{\mathrm{i}}, \operatorname{Veru}_{\mathrm{i}}, \mathrm{B}_{\mathrm{i}}, \operatorname{Vers}_{\mathrm{i}}, \mathrm{SID}_{\mathrm{j}}, \mathrm{Ts}\right)$ sent to the IoT server $S_{j}$.

12. witness(CS,U,auth_12, $\left.\operatorname{xor}\left(\operatorname{xor}(\mathrm{H}(\mathrm{H}(\mathrm{ID} \cdot \mathrm{H}(\mathrm{P})) . \mathrm{X}), \mathrm{H}(\mathrm{X})), \mathrm{NU}^{\prime}\right)\right)$

This pertains to the login and authentication phase of STEP 10, and the authentication server CS verifies whether the user $U_{i}$ is authenticated through the Vers $s_{i}$ value in the login request message $\left(\mathrm{UID}_{\mathrm{i}}, \mathrm{A}_{\mathrm{i}}, \operatorname{Veru}_{\mathrm{i}}, \mathrm{B}_{\mathrm{i}}\right.$, Vers $\left._{\mathrm{i}}, \mathrm{SID}_{\mathrm{j}}, \mathrm{Ts}\right)$ sent to the IoT server $S_{j}$.

13. witness(S,CS,auth_13, $\operatorname{xor}\left(\operatorname{xor}\left(\mathrm{NU}^{\prime}, \mathrm{NCS}\right), \mathrm{H}\left(\operatorname{xor}\left(\mathrm{SId}, \mathrm{NS} \mathrm{S}^{\prime}\right)\right)\right)$ )

This pertains to the login and authentication phase of STEP 12, and the IoT server $S_{j}$ verifies whether the authentication server $C S$ is authorized through the $C_{i}$ value in the mutual authentication message $\left(C_{i}, D_{i}, E_{i}, T s\right)$ sent to the authentication server $C S$.

14. witness(U,S, auth_14, $\left.\operatorname{xor}\left(\operatorname{xor}\left(\mathrm{NS} \mathrm{S}^{\prime}, \mathrm{NCS}\right), \mathrm{H}(\operatorname{xor}(\operatorname{xor}(\mathrm{H}(\mathrm{H}(\mathrm{ID} \cdot \mathrm{H}(\mathrm{P})) \cdot \mathrm{X}), \mathrm{H}(\mathrm{X})), \mathrm{NU}) \cdot \mathrm{H}(\mathrm{X}))\right)\right)$

This pertains to the login and authentication phase of STEP 15, and the user $U_{i}$ verifies whether the IoT server $S_{j}$ is authenticated through the $E_{i}$ value in the mutual authentication message $\left(E_{i}, T s\right)$ sent to the IoT server $S_{j}$. 


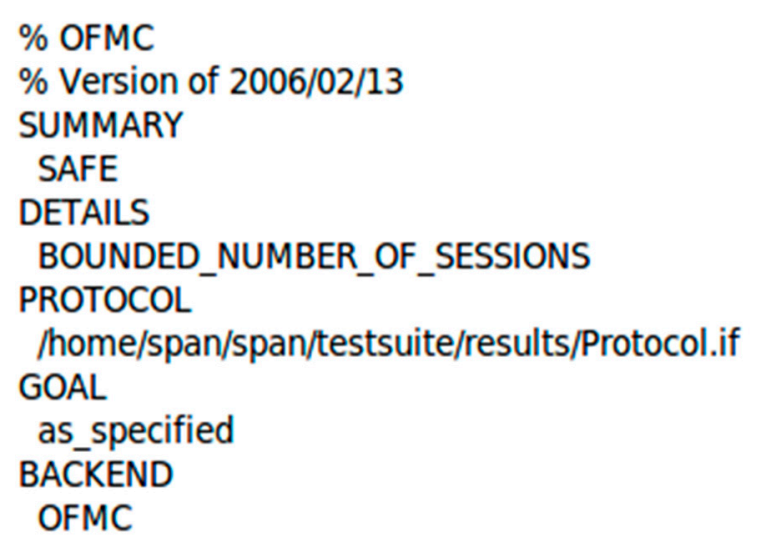

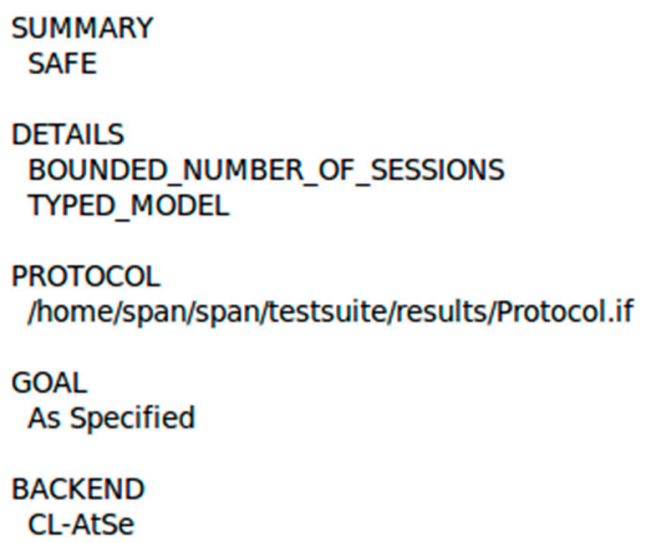

(b)

Figure 6. (a) Simulation result for the AVISPA tool under the OFMC model.; (b) Simulation result for the AVISPA tool under the CL-AtSe model.

In this study, the formal verification of the authentication protocol is performed through the OFMC and CL-AtSe models, as shown in Figure 5. and Figure 6. It can be seen that the $\boldsymbol{h}(\boldsymbol{x}), \boldsymbol{N}_{\boldsymbol{i 1}}, \boldsymbol{N}_{\boldsymbol{i 2}}$, and $\boldsymbol{N}_{i 3}$ values, which should be kept secret during the process of sending/receiving messages in the proposed authentication protocol, are not exposed to an attacker. Furthermore, through the verification performed by each entity, it was possible to confirm that the authentication performed between the user $\boldsymbol{U}_{\boldsymbol{i}}$, IoT server $\boldsymbol{S}_{\boldsymbol{j}}$, and authentication server $\boldsymbol{C S}$ was securely authenticated.

\section{Conclusion}

In recent years, as the scope of applications of IoT has broadened, the amount of data generated in IoT has become enormous, and the multi-server architecture has been utilized to manage this scenario efficiently. In a multi-server IoT environment, a user can manage and receive information collected by a sensor by connecting to a server via wireless networks from remote locations. However, if a malicious attacker accesses a communication network by exploiting a vulnerable authentication system, the system can be exposed to user impersonation and session key leakage attacks. Thus, a secure authentication protocol is required to prevent this.

Therefore, in this paper, we propose a secure authentication protocol to analyze and respond to security threats that may occur in a multi-server IoT environment. The proposed authentication protocol has been shown to be secure against user impersonations, session key leakage attacks, as well as various other attacks. The verification properties are specified by utilizing the formal specification language HLPSL. By using the formal verification tool AVISPA, the security of the required verification properties has also been verified through the results of our experiments.

The authentication protocol proposed in this paper is expected to be employed in applications such as key exchanges using smart cards, as well as applications in various other fields.

Acknowledgments: This work was supported by the National Research Foundation of Korea(NRF) grant funded by the Korea government(MSIP) (No. NRF-2014R1A2A1A11050818).

Author Contributions: Won-il Bae designed and verified the protocol. Wonil Bae then coordinated the writing of the manuscript, and contributed to the text. Jin Kwak conceived and supervised the work.

Conflicts of Interest: The authors declare no conflict of interest.

\section{References}

1. Pallavi Sethi and Smruti R. Sarangi. Internet of Things: Architectures, Protocols, and Applications. Journal of Electrical and Computer Engineering 2017, Volume 2017, pp. 25. 
2. R. Abdellatif, H. K. Aslan and S. H. Elramly. New real time multicast authentication protocol. International Journal of Network Security 2011, Volume 12, pp. 13-20.

3. C. C. Chang, H. L. Wu, Z. H. Wang, and Q. Mao. An efficient smart card based authentication scheme using image encryption. Journal of Information Science and Engineering 2013, Volume 29, pp. 1135-1150.

4. E. El-Emam, M. Koutb, H. Kelash and O. S. Faragallah. An authentication protocol based on Kerberos 5. International Journal of Network Security 2011, Volume 12, pp. 159-170.

5. D. He, J. Chen and J. Hu. Weaknesses of a remote user password authentication scheme using smart card. International Journal of Network Security 2011, Volume 13, pp. 58-60.

6. M. S. Hwang, S. K. Chong and T. Y. Chen. Dos-resistant ID-based password authentication scheme using smart cards. Journal of Systems and Software 2010, Volume 83, pp. 163-172.

7. Himanshu Mittal. Diffie-Hellman based Smart-card Multi-Server Authentication Scheme. Sixth International Conference on Computational Intelligence and Communication Networks 2014.

8. Tanmoy Maitra, SK Hafizul Islam, Ruhul Amin, Debasis Giri, Muhammad Khurram Khan and Neeraj Kumar. An enhanced multi-server authentication protocol using password and smart-card: cryptanalysis and design. Security and communication networks 2010.

9. Eun-Jun Yoon, Kee-Young Yoo. Robust Multi-Server Authentication Scheme. IFIP International Conference on Network and Parallel Computing 2009.

10. Vanga Odelu, Ashok Kumar Das, and Adrijit Goswami. A Secure Biometrics-Based Multi-Server Authentication Protocol Using Smart Cards. IEEE Transactions on information forensics and security 2015, Volume 10, pp. 1953-1966.

11. X. Li, Y. Xiong, J. Ma, W. Wang. An efficient and security dynamic identity based authentication protocol for multi-server architecture using smart cards. Journal of Network and Computer Applications 2012, Volume 35, pp. 763-769.

12. S. K. Sood, A. K. Sarje, and K. Singh. A secure dynamic identity based authentication protocol for multiserver architecture. Journal of Network and Computer Applications 2011, Volume 34, pp. 609-618.

13. Ruhul Amin, SK Hafizul Islam and Arijit Karati. Design of an Enhanced Authentication Protocol and Its Verification using AVISPA. Recent Advances in Information Technology 2016.

14. Sheikh Ziauddin, Bruno Martin. Formal Analysis of ISO/IEC 9798-2 Authentication Standard using AVISPA. Eighth Asia Joint Conference on Information Security 2013.

15. A. Armando, D. Basin and Y. Boichut. The AVISPA Tool for the Automated Validation of Internet Security Protocols and Applications. International Conference on Computer Aided Verification 2005, Volume 3576, pp. 281-285. 